\title{
Machine Vision System for Automated Visual Inspection of Tile's Surface Quality
}

\author{
Sunpreet Singh ${ }^{1}$ and Maninder Kaur ${ }^{2}$ \\ ${ }^{I}$ (ECE Deptt., Doaba Institute of Engineering \& Technology, Kharar, Punjab) INDIA \\ ${ }_{2}^{2}$ (ECE Deptt., Doaba Institute of Engineering \& Technology, Kharar, Punjab) INDIA
}

\begin{abstract}
Visual inspection of material's surface quality is an important quality determining step in industries dealing with surfaces as the prime feature of their product, for instance, plywood, metal, tiles, glass ware and many more. Primarily surfaces are inspected for smoothness and roughness quality. However, defects may arise in form of cracks, spots, holes, presence of excess material, flatness and shape of edges etc. A human inspection system can effectively challenge this task to a good accuracy. But with human system, there is an issue of fatigueness that arises over a long period of time. And in that case, the quality of inspection is biased towards either side. To avoid this biased inspection, a novel vision system based on image processing is proposed here. In the presented paper work, a case of tile industry has been tested out. The tiles surfaces are very sensitive to any of the defects as mentioned above. And a small defect on surface of a tile may lead to huge loss to the manufacturer. An automated visual inspection system inspects the tile's surface for any crack, hole, spot, presence of any foreign material or excess material. The objective of the proposed paper work is to develop an automatic machine vision system that could visually inspect the tile's surface to determine the quality as per the set standards. The work involves the extraction of defects from the surface using image processing techniques that are based on statistical properties of the defects observed on surfaces.
\end{abstract}

Keywords: Centre of Gravity (COG), Histogram Equalization, Bumps, Holes, Crack, Standard Deviation, Cornering

\section{INTRODUCTION}

$\mathbf{I}_{\mathrm{n}}$ tile's manufacturing industry, surface quality is of prime concern. An authentic determination of surface quality for each tile under production is a challenging task. Employing human personnel for this task, is one approach to achieve the target. However, the adherence to certain quality standards varies from person to person employed for the same task. And this variance leads to production of tiles of mixed quality. And surely, the human inspection system suffers from fatigueness when employed over a long time for the single task. Therefore, to get the same standard quality for each tile under production, an automatic visual system is required and that is free from any fatigueness.

\section{BRIEF LITERATURE SURVEY}

The tiles industrial sector has taken significant advantage of the strong evolution in the world of machine vision automation in recent years. All production phases have been addressed through various technical innovations, with the exception of the final stage of the manufacturing process [1]. This is still performed manually and is concerned with visual surface inspection in order to sort tiles into distinct

categories or to reject those found with defects and pattern faults. Therefore, traditional visual quality inspection performed by human inspectors has the potential to be replaced by computer vision systems [7]. Accurate automated inspection and classification can reduce human workloads and labor costs while increasing the throughput. A large amount of research has been carried out on automated inspection of tile surfaces [8-13], biscuit bake color [14], the color of potato chips [3], textile fabrics [11], food products [2] and wood [5]. The research effort expended upon the problem of objectively inspecting, analysing and characterising ceramic tiles is easily justified by the commercial and safety benefits to the industry [5] The late rise of the ceramic tile industrial sector means that there has been next to no attempts to automate final product quality inspection [10]. Many authors have discussed the detection of one type of fault only by analysis of the image intensity histogram [8]. The techniques range from small pin-hole and crack detectors for plain tiles, based on a set of separable line filters, through textured tile crack detectors based on the Wigner distribution and a co-joint spatial/spatial frequency representation of texture, to a colour texture defect detection algorithm which looks for abnormalities both in chromatic and structural properties of textured tiles.

\section{Methodology}

The scheme adopted to achieve the goal is summarized as: very firstly an image is grabbed using CCD camera and stored in bmp format with 8-bits gray level color scheme. The image is then subjected to noise removal algorithm. The noise free image is brought under threshold mode where the background is made white and object/patterns of interest are made black. This is termed as binarization of the image. After binarization, image is subjected to rotation independent cartesian coordinate system so that the statistical parameters to be computed are independent of any rotation. 
To compute the features of observed defects, the image is segmented based on connectivity criteria of pixels. Statistical parameters of the segmented image/defect are then extracted. The statistical features include, maximum and minimum radii in each quadrant, mean radius, intercepts on axes, normalized are, perimeter, and standard deviation of radii from centre of gravity of the image. The parameters are then normalized w.th respect to the mean radius to make them size independent. Fig. 1 shows the block diagram of the proposed system.

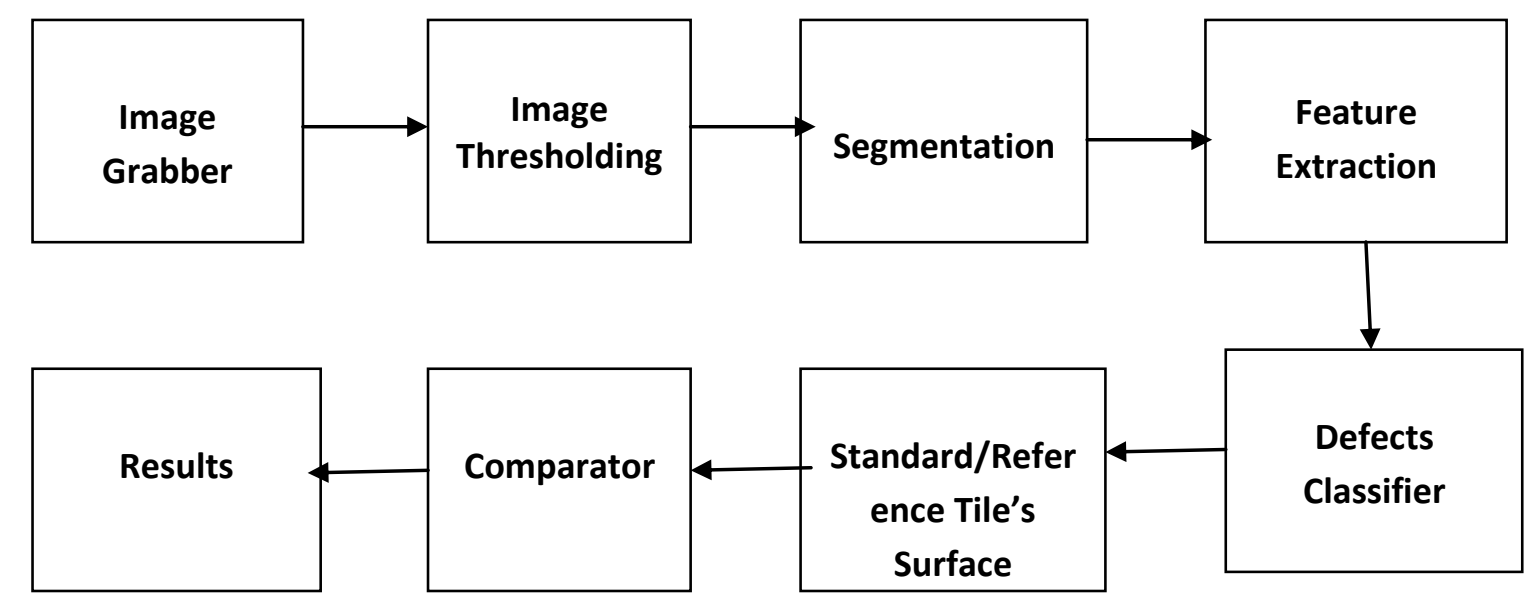

Fig. 1 Block Diagram of the proposed System

\section{IMAGE GRABBER}

Tile's surface image is grabbed by using the CCD camera from top of the surface from a distance adjusted so as to get the best possible view of the surface. Fig. 2 to 5 shows the tile's images. The tiles under test are of size $200 \times 200 \mathrm{~mm}$. For proper imaging, uniform lighting system is to be maintained to avoid any illusive defect by virtue of light reflection properties falling on surface.

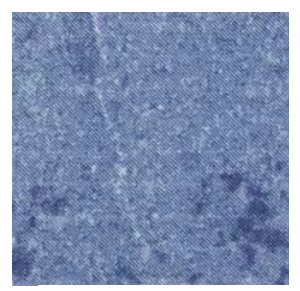

Fig. 2

Fig. 4

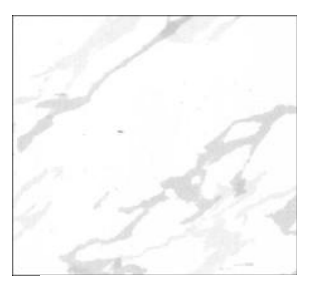

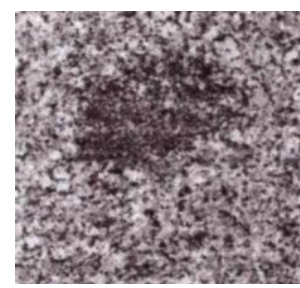

Fig. 3

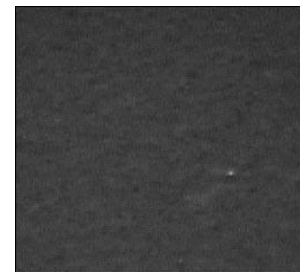

Fig. 5
While, applying image processing techniques for tiles surface defects identification, the original tile's design should always be kept in mind because many times tile's designs themselves create illusion with respect to the defects to be identified.

\section{IMAGE ENHANCEMENT AND THRESHOLDING}

Histogram equalization method is adopted to enhance the contrast of the tile's surface. Histogram equalization scheme works good in this case as the tiles surfaces contain few color segments by virtue of their design. Fig. 4 is an example here. Before applying the histogram equalization algorithm, image is converted to gray scale image using MATLAB command rgb2gray.

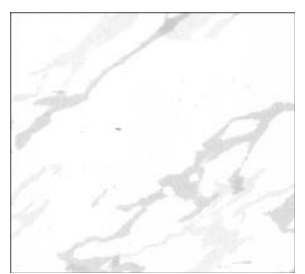

Fig. 6

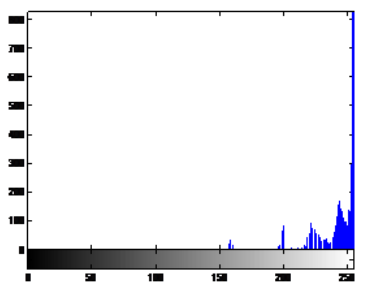

Fig. 8

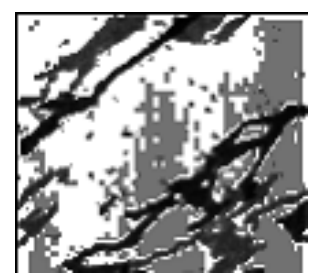

Fig. 7

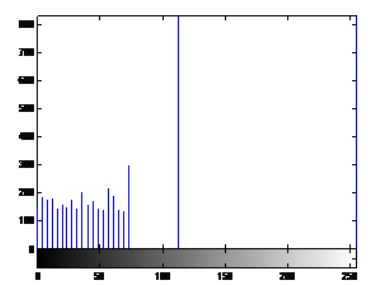

Fig. 9
Fig. 6 and 7 show the gray image and the image after application of the histogram equalization technique respectively. However, fig. 8 and 9 show the histogram of the above respective images. When applying the Otsu's algorithm over the enhanced image, we get a thresholded/binarized image as shown in fig. 10.

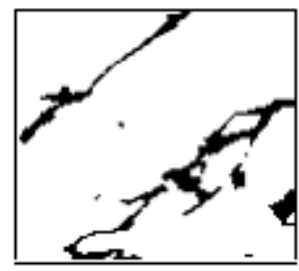

Fig. 10 


\section{SURFACE DEFECTS ClASSIFICATION AND GEOMETRICAL PATTERN RESEMBLANCE}

Normally, the defects observed on tile's surface are categorized according to the following:

- Cracks on surfaces $\rightarrow$ A crack is normally a linear identity and can be identified by a rectangle of very high aspect ratio. Further, if a crack is in the form of closed loop, then it can be identified by a circle with minimum standard deviation of radii. A crack may appear in the from of branch. In that case, the crack is identified by its spread along the dimensions. Scratches over the surface may also fall under this category of defects.

- $\quad$ Spots on surface $\rightarrow$ Spot on surfaces appear in the form of cluster of porous material. In that case, the pattern or design of the tile has high density or gradient of material at some particular location over the surface. This clustering is definitely different from the regular pattern of the tile surface. A spot may be identified by its degree of irregularity around its centre of gravity. Higher is the standard deviation of radii, more is the zig-zag shape of the spot.

- $\quad$ Bumps $\rightarrow$ may appear over the surface as a result of excess material fallen accidently. Many times, bumps gives shade change from regular to bump area. This can be identified by edge detection techniques using differentiation of color intensity method.

- Holes like defects $\rightarrow$ on tiles surface are rarely observed. However, the defects caused due to dent type shape may fall under this category. These may again be identified by edge detection techniques using differentiation of color intensity method. Also, these faults are primarily visible only on light plain tiles or lightly-textured tiles.

- Another important defect that arises very commonly is due to cornering. Tiles are very prone to corner/side breakage due to mishandling. This identification is done by using morphological operation on the image.

\section{SEGMENTATION}

The threshold image is now segmented based on bwlabel algorithm in MATLAB. Fig. 5 is taken as input image for segmentation. Below are the figures after segmentation using 8-connectivity of pixels and bwlabel function in MATLAB software. Fig. 11 and 12 shows the results of segmentation.

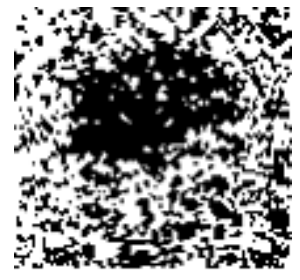

Fig. 11

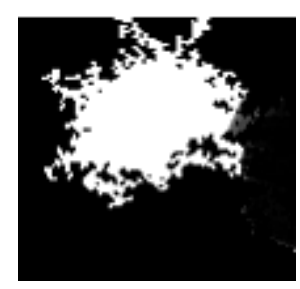

Fig. 12

\section{DEFECTS IDENTIFICATION}

Linear cracks are identified based on their figure aspect ratio. Once the crack line is segmented. Centre of gravity is computed by weight - averaging method ${ }^{[3]}$.

$$
\text { And } \quad \begin{aligned}
\mathrm{Gx} & =(1 / \mathrm{N}) \sum \mathrm{Xi} \\
\mathrm{Gy} & =(1 / \mathrm{N}) \sum \mathrm{Yi}
\end{aligned}
$$

Where $(\mathrm{Xi}, \mathrm{Yi})$ are the coordinates of the pixels on crack. After computing the COG, the crack is confined to a boundary box using the MATLAB function regionprops. Here, we can find out the figure aspect ration by computing the length and width of bounding box of the crack. It is observed that the figure aspect of a linear crack is of very high order, normally of the order of more than 300 .

A closed crack is identified by its standard deviation of radii from COG and euler number. For a closed circular shape, the euler no. is 1 and for open shape, the euler no. is 0 . The standard deviation for a closed circular crack should be less than 5. The Std. deviation of approximately 5 means that the crack under investigation is not fully circular closed but may vary to some extent. However, the circular shape of the crack is not very important. But the main thing is to identify a closed crack shape.

Spots on the surface are identified by their high degree of standard deviation of the radii from the COG. Bumps and holes like defects are identified by using the edge detection techniques. Edges may be identified by using sobel or prewitt filter.

Morphology of the tiles suggests the corner defects present in the tiles. Any irregularity in the straight edge of the tile will lead to morphological deformation. In the similar way, the corners may also be monitored. Morphological operations are methods for processing binary images also for gray scale images based on shapes. These operations take the binary and gray scale images as input, and return it as output. The value of each pixel in the output image is based on the corresponding input pixel and its neighbors. By choosing the neighborhood shape appropriately, you can construct a morphological operation that is sensitive to specific shapes in the input image.

\section{RESUlTS}

In the presented paper, the image was grabbed of size $256 \times 256$ pixel units using 3MPixel camera and from top of the tiles of size $200 \times 200 \mathrm{~mm}$. Fig. 2 shows the original image and below fig. 13 and fig. 14 show crack image and spot found in the two tiles after segmentation.

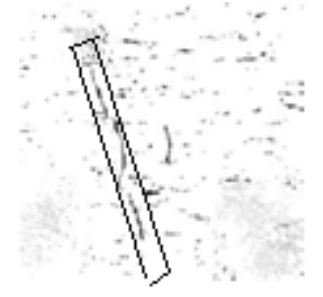

Fig. 13

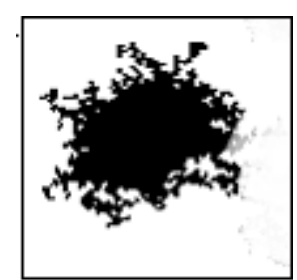

Fig. 14 
In fig. 13, crack is identified by the high figure aspect i.e. length to width ratio. The crack shape is bounded by the regionpros command in MATLAB and the figure aspect ratio is computed. However, the spot shown in fig. 14 is an irregular shape about its centre of gravity. High degree of standard deviation proves it to be a spot as not part of the original tile pattern. Standard deviation is given by:

$$
\mathrm{SD}=\sqrt{ }\left[\left(\mathrm{Ri}-\mathrm{R}_{\mathrm{M}}\right) 2 / \mathrm{N}_{\mathrm{p}}\right]
$$

Where $R_{i}, R_{M}$ and $N$ are the $i_{\text {th }}$ radius, mean radius and perimeter of the object (defect) respectively.

Similarly, excess material or dent in tile can be found by computing the size of the same. While observing the morphological properties of tile's image, corner defect may also be computed by observing the length and width of the entire tile.

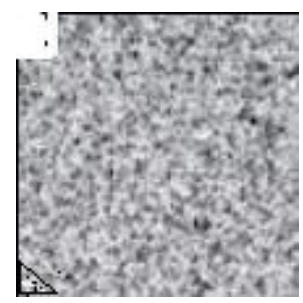

Fig. 15 (Corner Defect)

\section{Conclusion}

Tile's surface defects identification can be automated by installing a CCD camera on top of the production line over the conveyor belt, where the tiles arrive at certain regular interval. The camera along with the image acquisition and surface defects identification is interfaced with the PC to give results based on set criteria. More quality determining parameters can be identified and incorporated into the algorithm for better quality production of tiles. A report indicating the tile's surface may also be generated for the lot of production. And a statistical analysis can also be made regarding a particular trend obtained in the results. The analysis will give the manufacture an idea about the degree of contribution of an intermediate stage of the production line towards the quality deterioration.

\section{ACKNOWLEDGEMENTS}

We are thankful to Mr. Vikas Goel, Sr. Project Manager, CDAC, Mohali, Punjab for his valuable guidance and continuous support in making this paper.

\section{REFERENCES}

[1] W. Wen, and A. Xia, "Verifying Edges for visual Inspection Purposes." School of Mechanical and Manufacturing Engineering, School of Mathematics, The University of New South Wales, NSW 2052, Australia, July 1997.

[2] Mark E. Lehr and Keh-Shin Li, "Template basis techniques to Pattern recognition." University of California Riverside, Department of Statistics Riverside, California, USA, Vol. 2825, 1996.

[3] Yonghuai Liu and Macros Rodrigues, "A novel machine vision algorithm for a fast response quality control system." Department of Computer Science, the University of Hull, Hull, HU6 7RX, UK 2001.

[4] Stewart Coe, "Automatic tile inspection." Surface Inspection Limited, International Ceramics, Bristol, U.K., Issue 1, 2000.

[5] Costas Boukouvalas, Francesco De Natale, Josef Kittler, and Roberto Salgrai. "An Integrated system for Quality Inspection of tiles.” University of Surrey, Guilford, GU2-5XH, England, 1999.

[6] Martin Coulthard, "Measuring and Classifying Tile Shades." Chairman, Surface Inspection Ltd, Great Britain, Jan., 2001.

[7] Song K Y, Petrou M, and Kittler, "Texture crack detection." Machine Vision Applications, to appear Jan., 1995.

[8] Esko Herrala, "SIMON; Spectral Imaging for On line Sorting." Specim, Spectral Imaging Ltd., Finland, August 1998.

[9] Tony Lindeberg, "Edge Detection and Ridge Detection with Automatic Scale Selection." Department of Numerical Analysis and Computing Science, Royal Institute of Technology, International Journal of Computer Vision, S-10044 Stockholm, Sweden, Jan., 1996.

[10] C. Boukouvalas, J Kittler, R Marik, M Mirmehdi and M Petrou,"Ceramic Tile Inspection for Color and Structural defects." I.E.E.E. Transactions on Pattern Analysis and Machine Intelligence, vol. 14, no. 1, March 1998.

[11] Todd Reed and H. Wechsler, "Segmentation of textured images and gestalt organization using spatial / spatial-frequency representations." I.E.E.E. Transactions on Pattern Analysis and Machine Intelligence, vol. 12, no. 1 Jan., 1990.

[12] Vincent LEBRUN, "Quality control of ceramic tiles by machine vision," Flaw Master 3000, Surface Inspection Ltd. 2001.

[13] Simon Baker, "Design and Evaluation of Feature Detectors." Doctorof philosophy thesis in the Graduate School of Arts and Sciences, Columbia University, 1998.

[14] Richard Bridge, "Computer Image Processing." Tessella supportservices PLC, Oxon, England, Issue V1.R2.M0, June, 2003. 